\title{
Magnetic Shear and Nonpotential Energy Evolution of Solar Minimum and the Onset of Solar Cycle 23
}

\author{
J. Dun ${ }^{1,2}$ H. Zhang ${ }^{1}$ B. Zhang ${ }^{2}$ and R. Li $^{2}$ \\ 1.Beijing Astronomical Observatory, National Astronomical \\ Observatories, Chinese Academy of Sciences, A20 Datun Road, \\ Chaoyang District, Beijing 100012, China \\ 2.Yunnan Astronomical Observatory, National Astronomical \\ Observatories, Chinese Academy of Sciences Kunming, Yunnan, \\ 650011, China
}

\begin{abstract}
Using a 1995-1998 data set of vector magnetograms, the magnetic field flux, shear angle of the transverse field and nonpotential energy of active regions were calculated. The evolution of these parameters were analyzed together with time series of the solar monthly sunspot relative number and area to study their relationships in the ascending phase of solar cycle 23 . We find the magnetic flux and nonpotential energy have a good correlation with sunspot relative number and area. But the magnetic shear angle does not develop as above indices.
\end{abstract}

\section{Introduction}

It is generally believed that the energy released in solar flares is provided by the nonpotential components of the magnetic field in active regions. The angular shear(Hagyard et al. 1984) and the energy of source fields(Hagyard et al. 1981) are two of the parameters to denote the magnetic nonpotential character. The relationship between magnetic shear change and solar flares of individual active region has been extensively studied (Hagyard 1990; Wang 1992; Wang et al. 1994; Wang 1994; Chen et al. 1994;). However, the magnetic shear change of non-flare active regions is rarely studied. And the magnetic shear change related to the solar cycle has not been studied up to now. Solar activity is usually associated with an 11 year sunspot cycle. Do magnetic shear angle and nonpotiontial energy of active region vary as other solar activity indices? The vector magnetic field observation by the Solar Magnetic Field Telescope (SMFT), a vector magnetograph system (Ai \& $\mathrm{Hu} 1986$ ) installed at the Huairou Solar Observing Station (HSOS) of Beijing Astronomical Observatory, has provided a good chance to answer the question.

\section{Data and Method}

The vector magnetograph observes vector magnetograms at the photospheric line FeI $\lambda 5324.191 \AA$. In the measurement of the magnetic field in solar active regions, the integration of 255 frames and the $3 \times 4$ average are usually made so as to increase the signal to noise ratio. The view of the fields and the spatial resolution of the data are about $5.23^{\prime} \times 3.63^{\prime}$ and $2^{\prime \prime} \times 2^{\prime \prime}$ respectively.

Almost all active regions which were observed by SMFT at HSOS from 1995 to 1998 are chosen. For an active region, the observations during the period of two days it went through the solar disk center were chosen as possible. And 
for an active region, two magnetograms are chosen as possible in one day. But for some active regions, there was only one vector magnetograms for one active region. Thus about 700 vector magnetograms of 203 active regions are chosen.

Every magnetogram is calibrated by the method which has been described by $\mathrm{Ai}$ et al.(1982) and Wang et al.(1996). The $180^{\circ}$ ambiguity are resolved by the potential method (Gary \& Hagyard 1990). And for the vector magnetic fields which were observed away from the solar disk center, the method provided by Gary \& Hagyard (1990) were used to remove the projection effects.

The total magnetic flux $\Phi_{l}$ is

$$
\Phi_{l}=\sum_{i=1}^{n}\left|B_{l i}\right| \Delta a_{i}
$$

where $B_{l i}$ and $\Delta a_{i}$ are the longitudinal field and the area of the $i$ th pixel.

The average shear angle $\Delta \phi$ is defined as

$$
\Delta \phi=\left(\sum_{i=1}^{n}\left(\phi_{o i}-\phi_{p i}\right)\right) / n
$$

where $\phi_{o i}$ and $\phi_{p i}$ are the azimuth of of the $i$ th pixel of observed and potential transverse fields respectively.

The magnetic energy density of the fields is

$$
\rho_{o, p, s}=\sum_{i=1}^{n} \frac{B_{i}^{2}}{8 \pi}
$$

where $o, p, s$ are the observed fields, the potential fields and the source fields respectively and $B_{i}=\left|\mathbf{B}_{\mathrm{oi}, \mathbf{p i}, \mathbf{s i}}\right| . \mathbf{B}_{\mathrm{oi}}, \mathbf{B}_{\mathrm{pi}}$ and $\mathbf{B}_{\mathbf{s}, \mathbf{i}}$ are the observed, the potential and the source fields respectively. The source field is defined as $\mathbf{B}_{\mathbf{s}}=\mathbf{B}_{\mathbf{o}}-\mathbf{B}_{\mathbf{p}}$. And in above three equations, $n$ is the sum of the pixels in the active region.

Thus the total magnetic field flux $\Phi_{l}$, average shear angle $\Delta \phi$, the magnetic energy density of the observed and potential fields, and the nonpotential energy of the observed fields are calculated for every vector magnetogram.

\section{Result and Discussion}

To get the evolution of those parameters mentioned above, three steps are taken. First, the averaged values of the parameters of all magenetograms of an active region in one day are taken as the values of the active region at the day. Second, the averaged values of the parameters of all the active regions on the disk at the same day are taken as the values of that day. Then the averaged values of the parameters in a month are taken as the values of this month. Thus the time series of the parameters calculated above are obtained. The result is shown in Fig.1(b-f). The time series of two solar activity indices, monthly sunspot relative number and area, are also shown in Fig.1(a). The data of monthly sunspot relative number and area are taken from Chinese Solar-Geographysical Data (CSGD,1995-1998).

Comparing the evolution of the monthly sunspot relative number and area, with the evolution of the calculated parameters, we obtained the following results: 

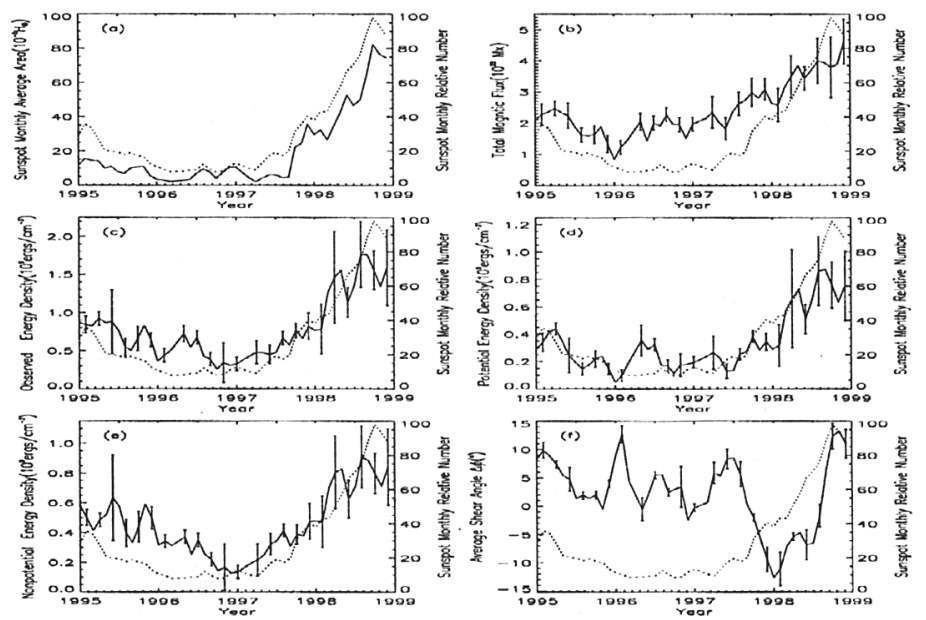

Figure 1. Time series of monthly values for sunspot area, total magnetic flux, observed energy density, potential energy density, nonpotential energy density, shear angle(solid lines) and sunspot relative number (dashed line) of active regions from 1995 to 1998 . Error bars denote the variations in different values of the same month.

1. The evolution of total magnetic field flux and the magnetic energy density of the observed, the potential and source fields of active regions have good correlations with the evolution of the monthly relative sunspot number and area

It shows that at the ascending of solar cycle, not only the total magnetic flux and energy of solar active regions are increasing, but also the nonpotial energy which carried by the new flux emerged below the photosphere is increasing.

2. The average shear angle does not show the same evolution as the above parameters. It does not change more than $15^{\circ}$ (in absolute value) from 1995 to 1998.

Acknowledgments. This work is supported by the Chinese Academy of Sciences Foundation of China and by the National Nature Science Foundation of China under grant 19791092.

\section{References}

Ai G., Li W. and Zhang H. , 1982, Acta Astron. Sinica, 23, 39

Ai G., and Hu Y., 1986, Pub. Beijing Astron. Obs. 8, 1

Chen J., Wang H., Zirin H., and Ai G., 1994, Solar Phys. 154, 261

Gary G. A., and Hagyard M. J., 1990, Solar Phys. 126, 21

Lu Y., Wang J., and Wang H., 1993, Solar Phys. 148, 119

Venkatakrishnan P., and Gary G.A., 1989, Solar Phys. 120, 235

Wang H., 1992, Solar Phys. 140, 85

Wang H., Ewell M.W., Zirin H., and Ai G., 1994, ApJ 424, 436

Wang J., 1994, Solar Phys. 155, 285

Wang T., Ai G. and Deng Y.,1996, Astrophysics Reports (Publication of the Beijing Astro. Obs.), 28, 31 